\title{
EIN VERGESSENES ARISTOTELESZEUGNIS
}

BEI dem seit 25 Jahren wogenden Streit um die Entwicklung der aristotelischen Gotteslehre, ${ }^{\mathbf{r}}$ in den auch mit wohl abwägendem Urteil Sir David Ross (Aristotle's Physics, $94 \mathrm{ff}$.) eingegriffen hat, ist eine Stelle aus Sextus Empiricus hyp. 3,2 I 8 nicht verwertet worden. Um aus dem Schwanken der Theologie in die skeptische $\dot{\epsilon} \pi \circ \chi \eta^{\prime}$ zu führen, gibt Sextus einen kurzen Katalog der Gottesauffassungen, wobei er wie 'Aetios' Doxogr. $297 \mathrm{aI} 3 \mathrm{ff}$. anlässlich eines ausführlichen Kataloges

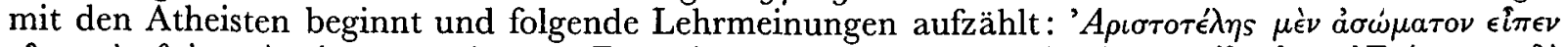



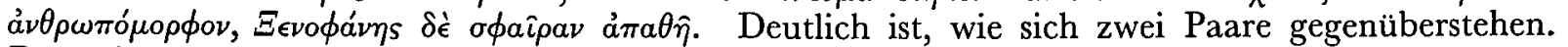
Der aristokratische Gott des Aristoteles, unkörperlich und uns entrückt, und der gemeine, körperliche und auch durch das Hässliche hindurchgehende des Stoikers, weiter der menschenförmige und der in idealer Kugelgestalt gedachte Gott. Die stoische Meinung ist gut wiedergegeben, höchstens dass in $\delta i \dot{\alpha} \tau \hat{\omega} \nu, \epsilon i \delta \epsilon \chi \theta \hat{\omega} \nu$ ein leiser polemischer Ton mitschwingt; ein frühes Zeugnis bietet Megasthenes, der in seinem Indienbuch für einen Zug der brahmanischen Philosophie die stoische



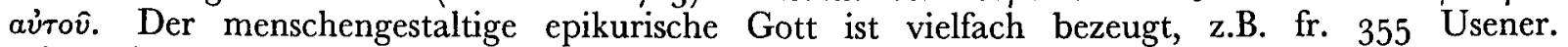

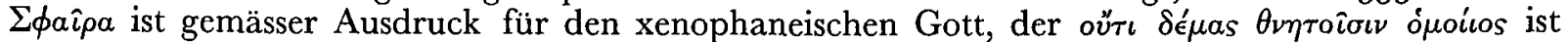
(B 23 Diels) und der säkularisiert in Parmenides' Kugel des Seienden erscheint (B 8, 43); Aristoteles fasste die Kugel des Xenophanes als Himmel (A 30). ${ }^{2}$ ' $A \pi a \theta \eta$ 's (auch A 35 Ende aus

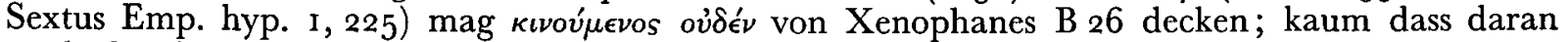



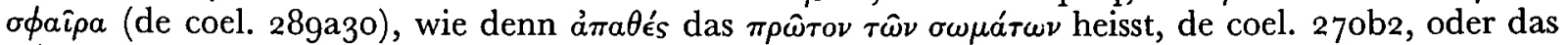
$\pi \epsilon ́ \mu \pi \tau o \nu \sigma \hat{\omega} \mu \alpha$, wie es Aristoteles vermutlich im Frühdialog de philosophia bezeichnete, ohne ihm einen Elementennamen zu geben,3 wenn er auch im volkstümlichen Namen 'Äther', den wir praktischerweise verwenden, die Ahnung des Richtigen erkannte (de coel. 270b22; Meteor. 339 b25

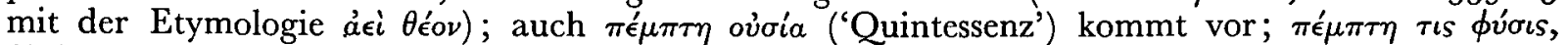

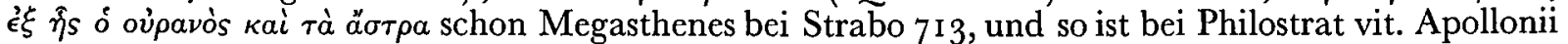

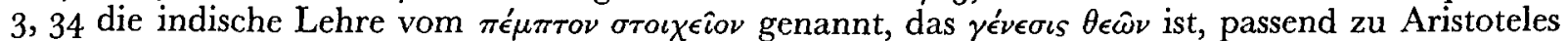

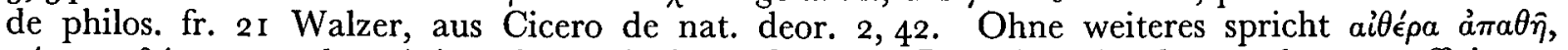
$\pi \epsilon^{\prime} \mu \pi \tau o \nu \delta \eta^{\prime} \tau \iota \sigma \hat{\omega} \mu a$ dem Aristoteles zu Aetios $336 \mathrm{a}_{5}$, und Ps.-Aristoteles de mundo $392 \mathrm{a} 5 \mathrm{ff}$. ist zu vergleichen; die Schrift mischt Aristotelisches und Poseidonisches.

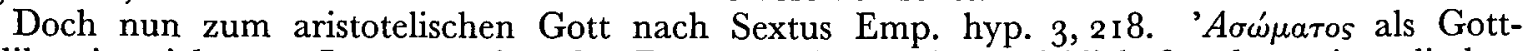
prädikat ist nicht nur Interpretation der Doxographie, sondern wirklich für den aristotelischen

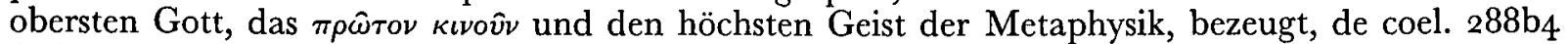


Es ist eine Stelle, deren Zusammenhang H. von Arnim Die Entstehung der Gotteslehre des Aristoteles I93I, I 8 ff. für einen spätern Einschub hält und so auch W. K. C. Guthrie, Class. Quart. 27, I933, I 70, der I67 allerdings fein bemerkt, die Aktualisierung eines Unvollkommenen durch ein Vollkommenes sei immer ein Grundgedanke des Aristoteles gewesen. Beide wollen nicht den 'Dyotheismus' einerseits von ätherischem Himmels-und Gestirngott, andererseits von $\pi \rho \hat{\omega} \tau o \nu$ kıvov̂v ákíntov dem ersten Entwurf von de coelo zuerkennen (gegen nachträgliche Ergänzung Ross, op. cit. 98, Cherniss op. cit. $5^{8} 4 \mathrm{ff}$.). Nun aber findet sich der Dyotheismus schon in der Frühschrift de philosophia, im berüchtigten Fragment $26 \mathrm{~W}$. aus Cicero de nat. deor. I, 33. Als aristotelische Götter werden aufgezählt einerseits 2. mundus, 4. caeli ardor (der Äther des Volksglaubens), anderer-

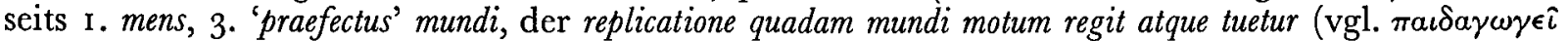
Plato Gesetze 897b). Replicatione ist schwer zu erklären; Cherniss 592 denkt mit von Arnim $4 \mathrm{f}$. an den Beweger der im Verhältnis zum Fixsternhimmel rückläufigen Planeten. Aber mundi motus muss die Vorwärtsbewegung des Kosmos im Ganzen sein, die im Kreise rückläuft; vgl. Chalcidius

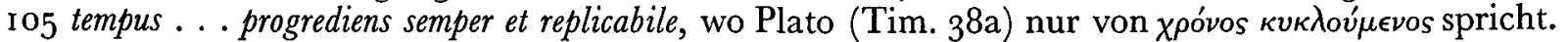
Der Epikureer wirrt absichtlich die verschiedenen Götter ineinander, spottet am Schluss darüber,

I Literatur bei $\mathrm{H}$. Cherniss, Aristotle's Criticism of Plato and the Academy, I 944, 584; A.-J. Festugière, La Révélation d'Hermès Trismégiste II, 1949, 259, I ; L. Alfonsi, Miscellanea Galbiati I, 195I, 7I, I (vgl. auch Hermes 8I, 1953, 45, 2 und schon Vigil. Christ. 2, 1948, $73 \mathrm{ff}$.$) .$

${ }_{2}^{2}$ Der menschenförmige Gott gegenüber dem jüdischen Gott als oủpavòs $\pi \varepsilon \rho \iota \varepsilon ́ \chi \omega \nu$ bei Hekataios von Abdera F gr Hist. 264 F6, 4 und Poseidonios $87 \mathrm{~F} 7 \mathrm{O}, 35$.
3 Richtig beobachtet im Altertum, vgl. Mich. Psellos, de omnif. doctr. I 3 I, S. 69 Westerink (= Cramer, Anecd.

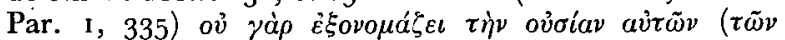
ä $\sigma \tau \rho \omega v)$ und so Cicero Tusc. I, 22 quintum genus vacans nomine; $4 \mathrm{I}$ quinta illa non nominata magis quam non intellecta

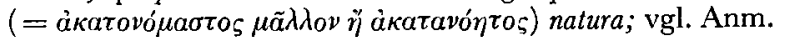

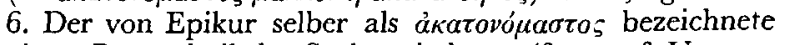
vierte Bestandteil der Seelenmischung (fr. 314 f. Usener; vgl. Lucrez 3, 242) ist fernzuhalten. 
dass der ảó́matos (sine corpore, carens corpore) mundus semper se movens sei.-Derselbe Dyotheismus wird von der Doxographie Theophrast zugeteilt, bei Cicero $\$ 35$ modo enim menti divinum tribuit principatum, modo caelo (und auch Gestirnen), Clemens von Alexandria protr. 66, $5 \pi \hat{\eta} \mu \dot{e} v$ ov่pavóv,

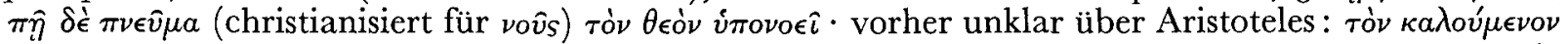

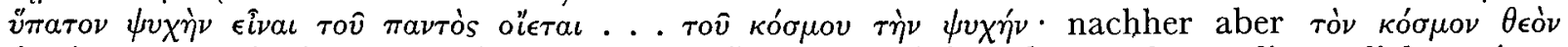

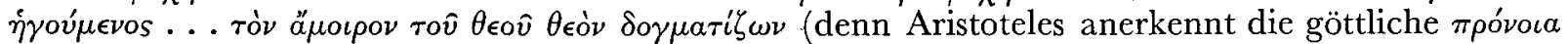
nur in der obern Region vom Monde aufwärts an)-ähnlich wie Cicero über den Widerspruch spottet, dass der Äther Gott sein soll, nur ein Teil der anderwärts als Gott bezeichneten Welt.

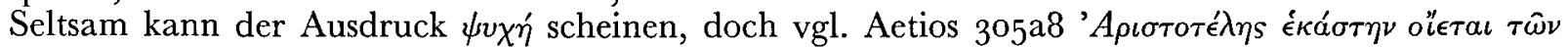

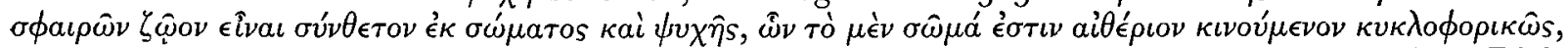

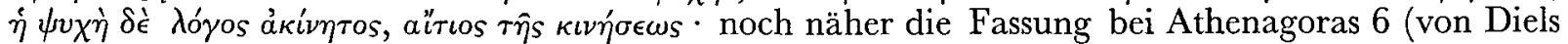

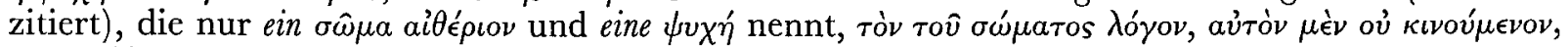

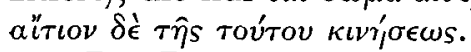

Der Dyotheismus kam schon in Aristoteles' Frühschrift vor, und falls Theophrast den Namen $\pi \epsilon ́ \mu \pi \tau o \nu ~ \sigma \hat{\omega} \mu a$ gebrauchte (nach fr. 2 I, Doxogr. 493, 8), schloss er sich einer frühern Ausdrucksweise des Aristoteles an.4 Wenn de coelo thematisch in der Hauptsache nur den sichtbaren Gott behandelt, so wird deswegen nicht eine alte Gedankenschicht des Aristoteles erreicht; auch die Eudemische Ethik, ganz dem praktischen Leben hingeneigt, verzichtet auf die Darstellung des

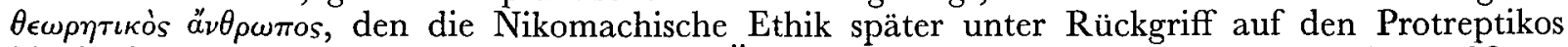

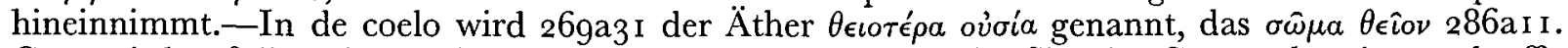
Gern wird auf die volkstümliche Auffassung verwiesen, dass der Sitz der Götter oben ist, $27 \mathrm{ob}_{5} \mathrm{ff}$., 278b I 5, 284a I I f., die letzte Stelle beweist, dass nicht an den ersten bewegenden Gott gedacht ist;

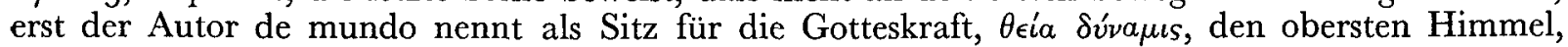

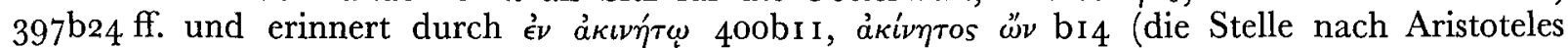

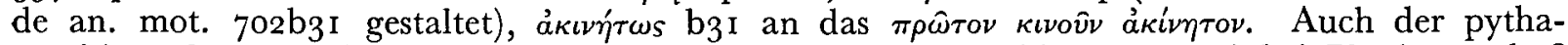
goreisierende Autor (Agatharchides nach Immisch, Sitzber. Heidelberg I919) bei Photios $439 \mathrm{~b}_{2} 6$

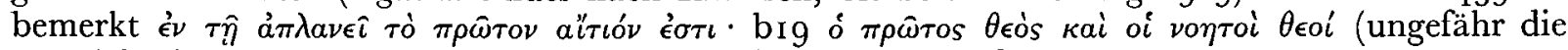

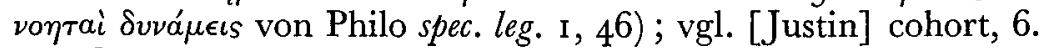

Die platonische Himmelsbeseelung, die offenbar in De philosophia Geltung hatte, ist auch in De coelo nicht ganz verschwunden, 285a29; 292a I 8 (auch von Simplicius in de coelo 378 , I $2 \mathrm{f}$. zitiert), und Theophrast verlangt die Himmelsseele, Metaph. $5^{\mathrm{b} 2}$ und bei Proklos in Tim. 2, I22,

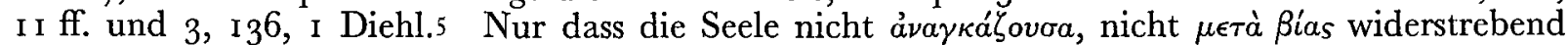
die Elemente stösst (Simplicius 378, $35 \mathrm{ff}$; Ross op. cit. 98), wird de coel. 284 a22 ff. dargelegt, in einem Stück, das, wie W. Jaeger, Aristoteles 320 sah, der Jugendschrift nahe steht, in der zwar noch nicht dem Äther von Natur-Natur im Sinn der terrestrischen Physik gebraucht-die kreisläufige Bewegung zugeschrieben wird, aber auch die Bía (vis) abgestritten wird, derart dass $\pi \rho \circ a i \rho \epsilon \sigma \iota s$ (voluntas) den vermutlich ruhig schwebenden Äther in Bewegung setzt; fr. 2 I aus Cicero de nat. deor. 2, 44 .

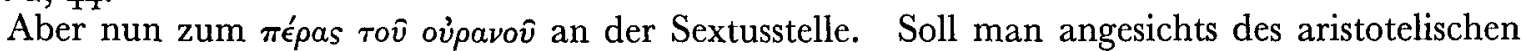

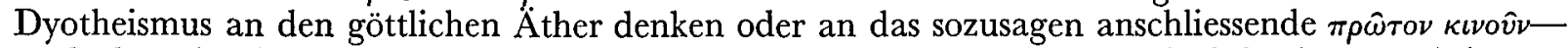

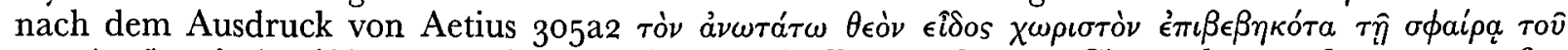

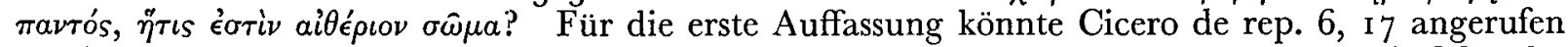
werden, der die Fixsternsphäre summus deus arcens et continens ceteros deos nennt. M. Messala, cons. 53 v. Chr. also zur Zeit, als Cicero an seinem Staatswerke schrieb, erklärte nach Macrob Sat. I, 9, I4 den weltschaffenden Ianus (Aí́v nach Lydus de mens. 64, I 2 Wünsch) als den Gott, der die auseinanderstrebenden Elemente copulavit circumdato caelo; quae vis caeli maxima duas vis dispares colligavit. Gott und Himmel scheinen hier dieselbe Aufgabe zu haben. ${ }^{6} \mathrm{Zu}$ vergleichen ist, was

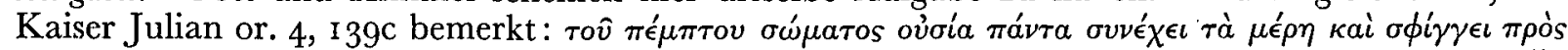

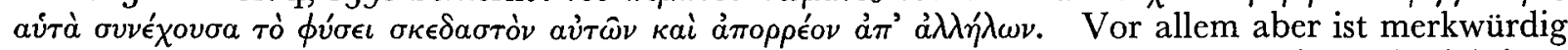
eine Notiz des Hippolyt elenchos 7, I 9, 2 ff., der den Ketzer Basileides des Aristotelismus bezichtigen

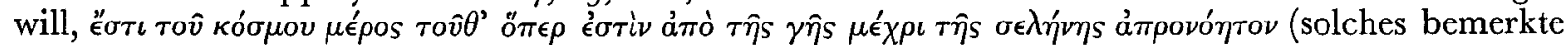

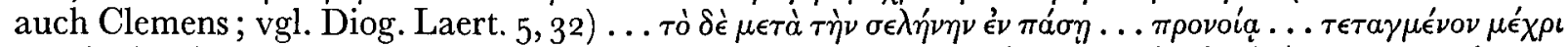



4 Theophrast benutzt auch nicht die späteste Fassung der aristotelischen Ethik; vgl. Hermes 69, 1934, 354, 376.

5 Über die theophrastische Definition ( $\varepsilon^{2}$ évioıs) der

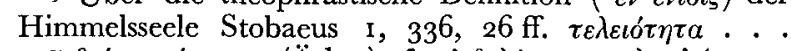

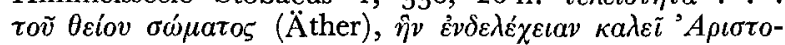
$\tau \varepsilon \dot{\lambda} \lambda \zeta$. Dagegen polemisiert bei Julian or. 5, I62b

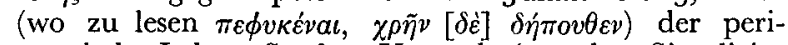
patetische Lehrer Strabos Xenarch (von dem Simplicius

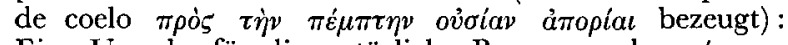
Eine Ursache für die natürliche Bewegung des $\pi \varepsilon \dot{\mu} \mu \pi \tau \nu \nu$ $\sigma \tilde{\omega} \mu a$ sei nicht zu suchen, das verstosse gegen einen Satz gerade Theophrasts; er ist uns erhalten Met. 9 b 2 I und bei Proklos in Tim. 2, 120, 7 ff. Zur Himmels-beseclung vgl. noch Arius Did. Dox. 450, I9; [Plutarch] poes. Hom. I05; Alexander v. Aphr. quaest. I, 25.

6 Vgl. Clem. Rom. Recogn. 8, I5 Aristoteles (de philos, fr. 27 Ross) etiam quintum introducit elementum... ákatovó $\mu \alpha \sigma \tau o v . .$. sine dvbio illum indicans qui in unum quattuor elementa coniungens mundvm fecerit. 


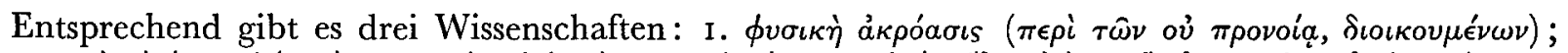

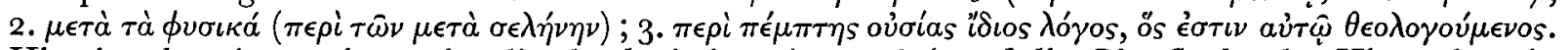
Hier ist also eigenartigerweise die theologische $\pi \epsilon^{\prime} \mu \pi \tau \eta$ ov $\sigma^{\prime} \alpha$ auf die Oberfläche des Himmels, sein $\pi \epsilon ́$ pas, beschränkt. Die Theorie ist nicht einfach eine Missgeburt aus dem Gehirn des Hippolyt.

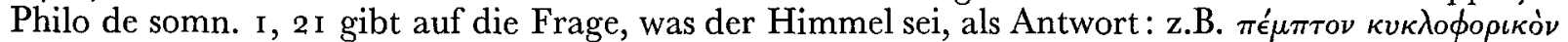

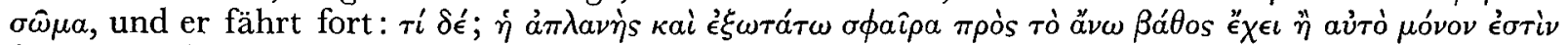

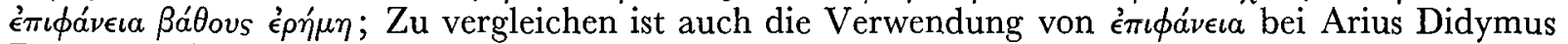

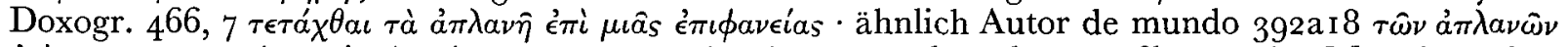



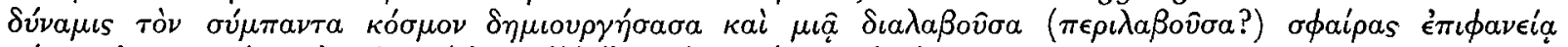

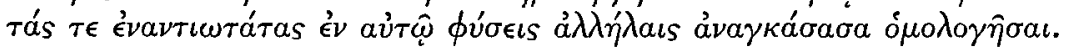

Der Dreietagenbau bei Hippolyt lässt sich in gewisser Beziehung auf Aristoteles zurückführen. Dieser nennt die zwei obern Etagen (Fixstern-bezw. Planetenhimmel) ausdrücklich de coel. $278 \mathrm{~b}$ i I ; ähnlich 'Agatharchides' 44Ia3. Verdächtig ist die Dreistufung bei Philolaos A I6: ö $\lambda \nu \mu \pi$ os (wo

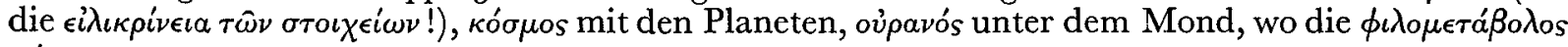
$\gamma^{\prime} \dot{v} \in \sigma t s$.

Aber es gibt noch eine andere Dreietagenordnung im Umkreis des Aristoteles und vielleicht hilft sie uns weiter. Von unten nach oben: terrestrische Sphäre, Himmelssphäre im ganzen und-



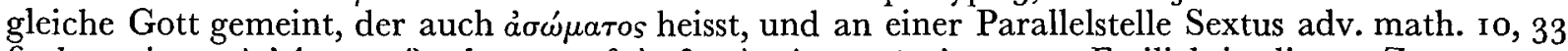

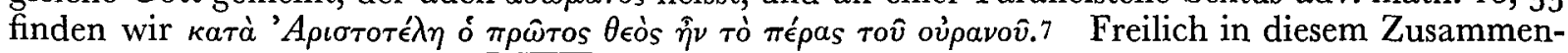

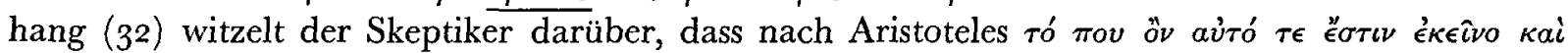

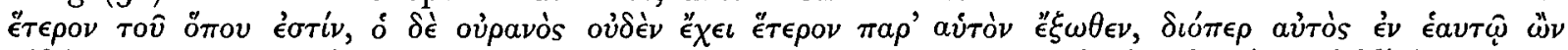

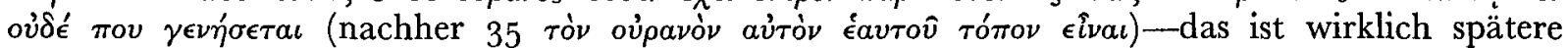

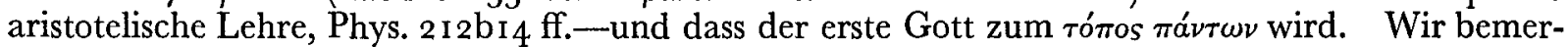
ken nur im Vorbeigehen, dass eine solche Lehre Philo von Alexandria tiefsinnig vorgekommen ist,



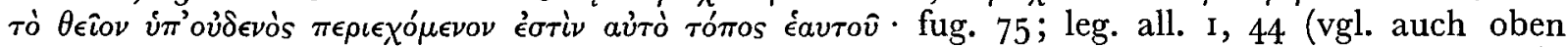


der äussersten $\pi \epsilon \rho \iota \phi \varphi^{\prime} \rho \epsilon \iota \alpha$ des Fixsternhimmels unterzubringen-was bis zu einem gewissen Grade die seltsame Ansicht bei Hippolyt erklären könnte-so Alexander von Aphrodisias bei Simplicius in

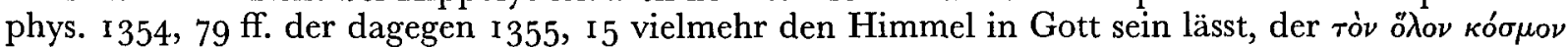

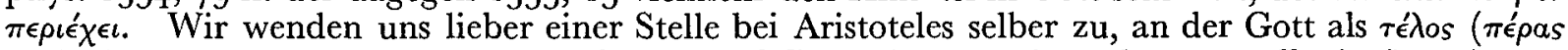



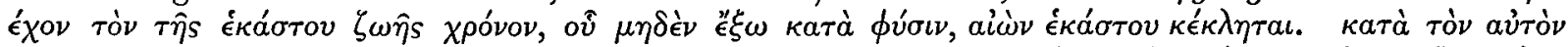

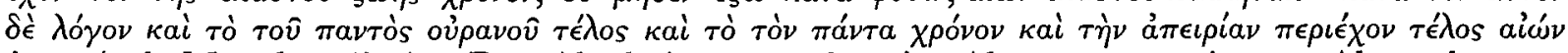
$\dot{\epsilon} \sigma \tau \iota \nu$ (vgl. Messalas Aíúv). Der Abschnitt war schon im Altertum umstritten. Alexander von Aphrodisias (bei Simpl. in de coel. 290, I ff., 287, I9 ff.) wollte das Ganze im Sinne von Arnims auf das $\kappa v \kappa \lambda \circ \phi о \rho \eta \tau \iota \kappa \dot{\nu} \sigma \hat{\omega} \mu \alpha$, Hauptgegenstand von de coelo, oder auf die Fixsternsphäre zielen lassen

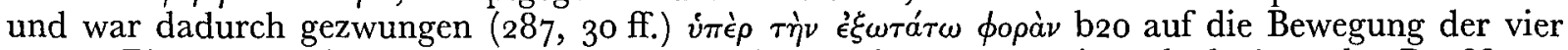

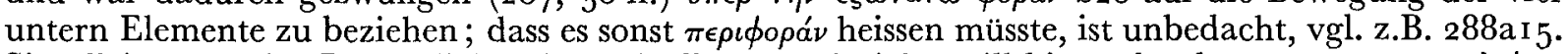

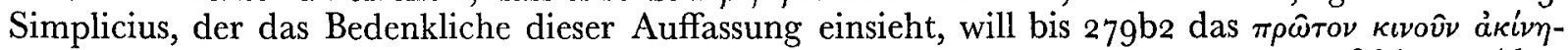

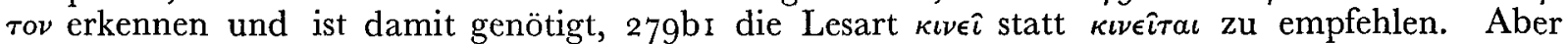

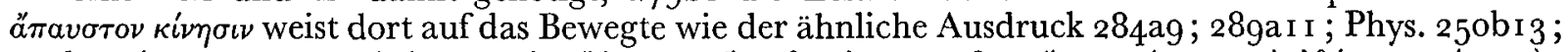

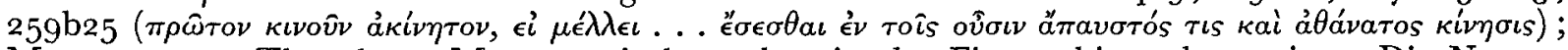
Met. Io72a2I; Theophrast Met. 5a3; insbesondere ist der Fixsternhimmel gemeint.-Die Neuern machen entweder bei de coel. 279a22 (Guthrie i68, Ross 97) oder bei 279b (Cherniss 588, Gigon in der Übersetzung im Artemis-Verlag i 950, 22) den Übergang zum bewegten Himmel. Auch



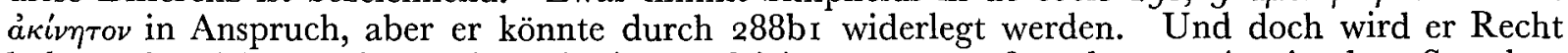

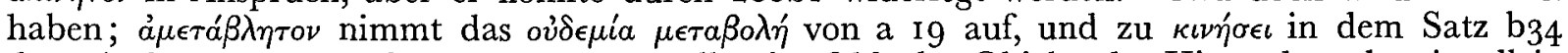
der mit de an. mot. 70ob34 zusammenzustellen ist, fehlt das Objekt, der Himmel, und so ist allein

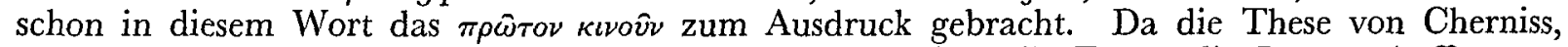
279a 8-35 als grosse Parenthese zu fassen, nicht befriedigt-die These, die Jaegers Auffassung, das Stück auf die Schrift de philosophia, die ja auch zitiert wird, zurückzuführen, stützen könnte-, wird man versuchen, $279 \mathrm{bI}$ direkt anzuschliessen: nun, wie zu $\kappa \iota v \eta^{\prime} \sigma \epsilon \iota$ das Objekt fehlte, so fehlt $\mathrm{zu} \kappa \iota v \epsilon i \tau \alpha \iota$ das Subjekt ('es gibt entsprechend eine unaufhörliche Bewegung'); vgl. SchwyzerDebrunner, Griech. Grammatik 2, 239 Nr. 4.

Aion, als überzeitliche, alle zeit-einschliessende (vgl. Phys. $22 \mathrm{Ib} 2 \mathrm{ff}$.) Ewigkeit, ewiges Leben

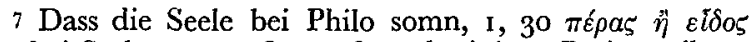
ist, bei Stobaeus I, 363 , Ig f. nach einigen Peripatetikern VOL. LXXVII

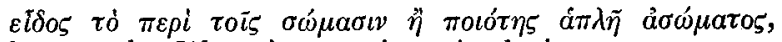
hat nur den Wert einer gewissen Analogie. 
gefasst, umhüllt den Himmel-denn ausserhalb des Himmels gibt es keinen zeitmessenden bewegten Körper-, und da Gottes Leistung selber Unsterblichkeit, ewiges Leben ist und um der Leistung, also des ewigen Lebens willen existiert, ist das von ihm abhängige $\theta \epsilon \hat{\imath} o \nu$ ewig bewegt (286ag), schliesst alle untergeordnete Bewegungen wie ein tépas ein (284a4). Die Bewegung des ersten

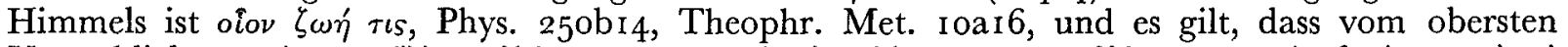


$\zeta \hat{\eta} \nu$, de coel. 279a28; vgl. Met. I072bi4; de an. mot. 70oa6; Theophr. Met. 4 bi 5 .

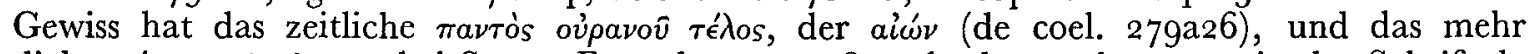

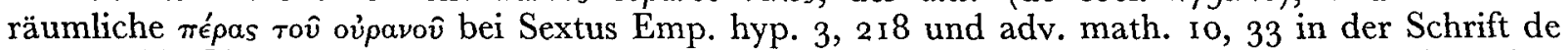

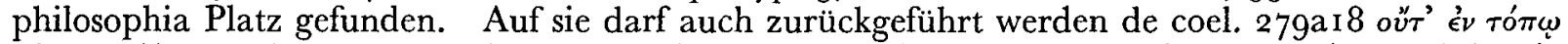

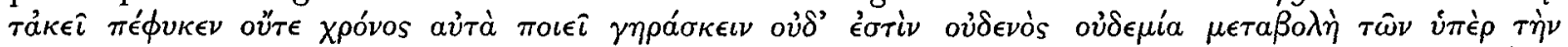

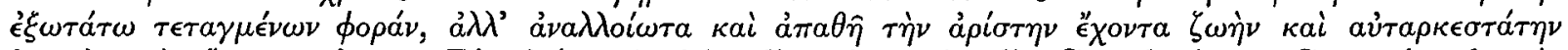

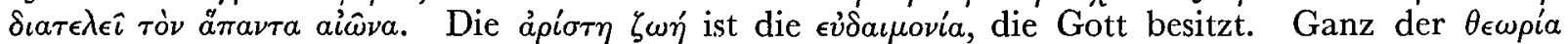

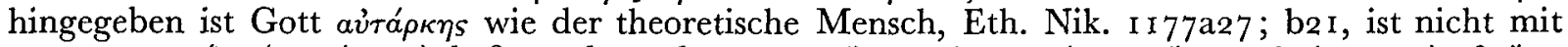

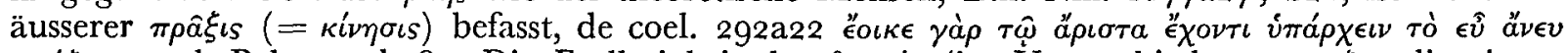

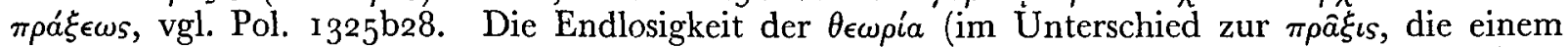

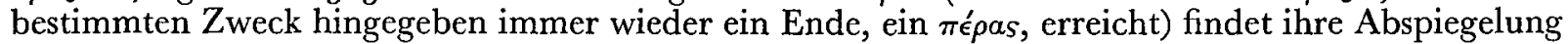
in der ewigen Bewegung des dem höchsten theoretischen Gotte nächsten Himmels. Gott ist quietus et beatus nach de philosophia fr. 26, wo der übermittelnde Epikureer wieder mit der Paradoxie


Lebens in der Akademie (Protrept. bei Jambl. Protr. 58, I3 ff. Pistelli) hat Aristoteles früh die geistige é $\nu^{\prime} \hat{e}_{\gamma} \gamma \iota \iota$, die er nach der Abwendung von der Ideenwelt über die körperliche Welt gesetzt

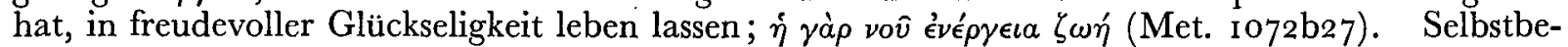


gibt. Die der Materie entblössten $\epsilon i \delta \delta \eta$ der Welt, wie die Metaphysik des Aristotles sie ergründet (vgl. Met. 983a9), erfüllen den Geist Gottes (wie etwa der Architekt ein Haus ohne Materie in seinen Sinn fasst, Met. I032bi3 ; I034a24; de gen, an. 730bi 4 ff.). Nicht ausgesprochen ist, dass auch alle "̈ $v v \lambda \alpha \epsilon^{\prime \prime} \delta \eta \eta$ göttliche Bestimmtheit in der durch den Umlauf der Planetensphären angeregten Weltveränderung erstreben.-Wir berührten den Dreietagenbau im Kreise des Aristoteles.

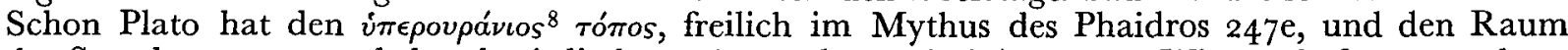
der Sternbewegung und den der irdischen $\gamma^{\prime} v^{\prime} \in \sigma \iota s$, denen drei Arten von Wissenschaft entsprechen, geschieden. Das wirkt bei seinen Schülern nach. Philipp von Opus in der Epinomis feiert hauptsächlich das mittlere Stockwerk der Astronomie die gleich der $\theta \epsilon o \sigma \epsilon \epsilon \epsilon \iota a$ ist. Bei Xenokrates gibt es (fr. 5 Heinze) die oberste Stufe des voๆróv, das Idealzahlenreich, und den Bereich des

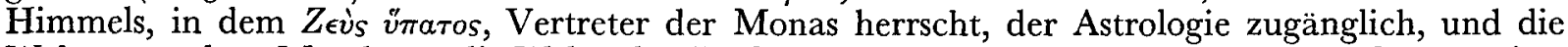
Welt unter dem Mond, wo die Weltseele, die Göttermutter, Vertreterin der aoristos Dyas regiert (fr. I5. I8). Ähnlich unterscheidet Aristoteles (Met. I06ga3o ff.) nach dem Fall der Ideenlehre die ákivntos oủoía des Geistes-nie konnte die höchste Norm fehlen, 9 nie konnte es also im von

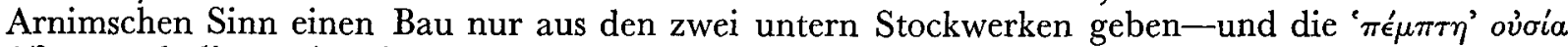
ảiócos und die oúcía $\phi \theta \alpha \rho \tau \grave{\eta}$. Die entsprechenden Wissenschaften sind Metaphysik (Theologie), Himmelsphysik (welcher Mathematik hilft) und terrestrische Physik (mit Meteorologie). Die drei aristotelischen Stockwerke finden sich z.B. bei Philo quaest. gen. 4, 8, danach Lydus de mens. 28, $10 \mathrm{ff}$. Der sog. Onatas bei Stobaeus I, 49, 2 ff. Wachsm. stellt dem $\pi \rho \hat{\omega} \tau$.

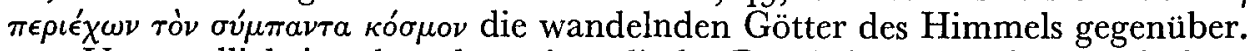

Verständlich ist, dass der aristotelische Dyotheismus - bei Plato fehlt er, da er die der Seele und dem Geiste vorgeordneten Ideen nicht als Götter bezeichnete-Bedenken erregen konnte, und dass Versuche gemacht wurden, die Geistsubstanz dem göttlichen Himmel oder insbesondere der Fixsternsphäre (so auch nach Arius Did. Dox. 450, 15) zuzuordnen. Ein später Gegner des Aristoteles, der Philosoph Attikos, meint (bei Euseb. praep. ev. I5, 7,6) maliziös, das $\pi \dot{\epsilon} \mu \pi \tau o v \sigma \hat{\omega} \mu a$

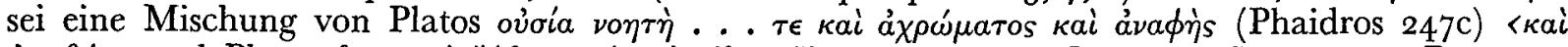

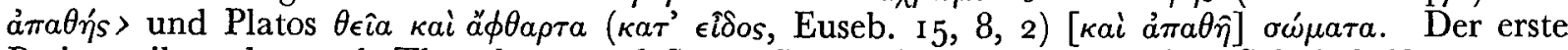
Peripatetiker, der nach Theophrast und Strato Kenntnis der aristotelischen Schulschriften verrät und auf Okellos (um roo v. Chr.) gewirkt hat, Io Kritolaos (und sein Schüler Diodor), lässt Gott

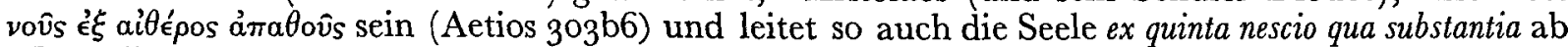
(Tertullian de an. 5; Macrob in somn. Scip. I, I4, 20). Nach Stobaeus I, 366, 25 fassten einige

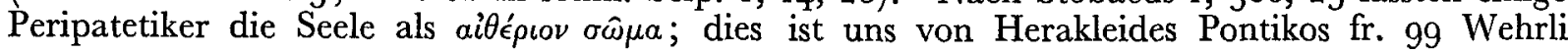

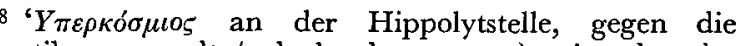
Gnostiker gewandt (vgl. elenchos 7, 23, 2) zeigt, dass der dortige Etagenbau nicht ganz unabhängig vom jetzt betrachteten ist. Vgl. auch Vita Aristotelis Marciana

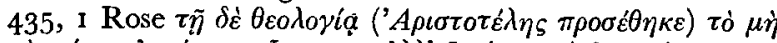

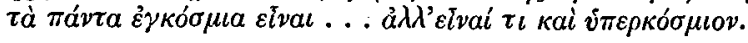

9 Auch die kreisende Bewegung der Gestirngötter oder des Äthers verrät eben die Wirkung einer geistigen $\tau \alpha \xi_{l \zeta}$, Met. 1025b25; $1060 a 26$.

so Gnomon 1926, 590 ff. Bei Okellos 37 Harder auch Andeutung des $\ddot{A}$ thers unter Beseitigung des Dyotheismus. 
überliefert. Der nämlichen Auffassung folgte Antiochos von Askalon, der in seiner akademischperipatetischen Einheitsphilosophie auch seine stoischen Sympathien zur Geltung brachte. Sie liegt vor bei Cicero Acad. I, 26 (quintum genus e quo astra mentesque, ähnlich Philo heres 283; aber anders der alte Zeuge Megasthenes, der den Geist weglässt), 39; de fin. 4, 12, wohl auch 36 (animus in quodam genere corporis) und 2, II4. Unbestimmbar ist der Übermittler für Ciceros Tusculanen, wo weniger die Verstofflichung des Geistigen als die Vergeistigung der quinta natura vorliegt: 1 , 22 $2^{\text {II }}$ im Vergleich mit Plato Gesetze 897 a und 66 mit der pathetischen Hervorhebung der Freiheit, Körpergelöstheit des Göttlichen (ähnlich dem, was Plutarch de parte aut facult. animi 5, VII I5, I $5 \mathrm{ff}$. Bernardakis von der Seele bemerkt). Wir sind gehalten, Cherniss 6o r f. zu folgen und solche Lehren nicht der Schrift de philosophia zuzuweisen trotz neuer Fürsprache bei Festugière 255 f. (mit geistvoller Begründung) und Alfonsi (vgl. oben Anm. I).

\section{Universität Bern.}

WiLly TheIleR

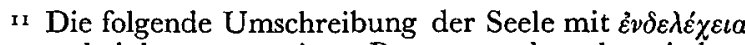
passt scheinbar zur ewigen Bewegung der platonischen Weltseele. Theophrast hat nach seiner Anm. 5 wieder-


gerade nicht gebraucht, und auch Aristoteles, der

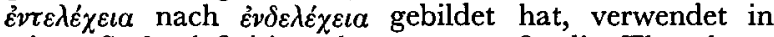
seiner Seelendefinition de an. 412a28, die Theophrast

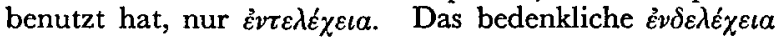

kann beim gemeinsamen Vorfahr von Cic. Tusc. I, 22; Philo somn. I, 3o, der das ảó́ $\mu \alpha \tau o \nu$ betont, und Macrob in somn. Scip. I, I4, 19 auf das selbe Missverständnis zurückgeführt werden, das bei Arius Did. Dox. 448, 20

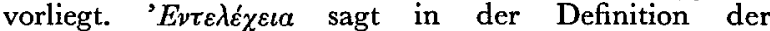
menschlichen Seele Xenarch nach Aetios $388 \mathrm{bi6}$; vgl. Simplicius in de coelo $3^{80}, 16$. 